\title{
“嫦娥一号”对月球新生环行山表面热辐射 “热点”与 “冷点” 昼夜变化的观测
}

\author{
宫晓蕙，金亚秋* \\ 复旦大学波散射与遥感信息重点实验室, 上海 200433 \\ * 通信作者. E-mail: yqjin@fudan.ac.cn
}

收稿日期: 2012-03-15; 接受日期: 2012-06-27

国家自然科学基金 (批准号: 41071219, 60971091) 资助项目

\begin{abstract}
摘要 20 世纪 60 年代由地基观测的月球表面红外辐射扫描图像发现月蚀过程中月表面有大量的 异常“热点”, 而在“嫦娥一号” ( CE-1) 对月球表面微波辐射亮度温度 ( Tb) 观测中, 这些红外辐射 “热 点” 的地方恰是夜间微波热辐射的 “冷点”. 本文以辐射传输理论分析, 解释了这些热辐射昼夜变化 很大的异常现象. 为比较月表面昼夜辐射温差的变化, 选取了在相近纬度上两种典型的环形山区域: 一是表面有大量离散岩石块分布的代表新生的环形山(第谷 Tycho Crater), 另一是经历了长期陨石 冲击粉碎与月质演变的不再有大量岩石块分布的老年环形山 (莫洛利卡斯 Maurolycus Crater). 给出 了这两环行山区域的 CE-1 微波 $\mathrm{Tb}$ 数据的昼夜变化, 并与美国 2009 年月球轨道勘测器 “先知者” (Diviner) 探测对应的红外辐射做了对比. 以月表 3 层模型的辐射传输为基础, 从 $\mathrm{CE}-1 \mathrm{~Tb}$ 数据反演 了该两区域月壤介质层物理温度的昼夜变化及其分布将月表面物理温度昼夜变化与红外观测结果 做了比较. 可以看出, 月表面热辐射与物理温度的昼夜变化分布, 及其 “热点”与 “冷点”的变化与月 表面新生环形山大量分布的高热传导高热惯性岩石块丰度 (abundance) 分布密切相关.
\end{abstract}

关键词 嫦娥一号 $\mathrm{Tb}$ 冷热点 热辐射昼夜变化 微波与红外热辐射 岩石丰度分布 微波 红 外辐射 行星表面分析

\section{1 引言}

具环行山分布的月球表面红外与微波热辐射观测在近半个世纪以来一直是受关注的研究领域. 月 表面热辐射的变化可以提供有关月球物理的许多科学知识, 如月球地貌、月球地质、分层结构、介电 特性等. 早在上世纪 60 年代, 用地基红外辐射扫描图像观测到月蚀期间月表面有大量非均匀分布的 辐射 “热点” [1]. 后来, 由月球轨道勘测器 LRO (lunar reconnaissance orbiter) 计划的 Diviner IR 红外 辐射数据显示具有大量离散分布的岩石块的新生环形山, 在夜晚的红外辐射温度要比周围环境高, 看 作是异常的热辐射现象 ${ }^{[2]}$.

我国 2007 年 10 月 24 日成功发射了第一颗探月卫星 “嫦娥” 1 号 (CE-1)，作为 “嫦娥” 1 号的备 份星, 具有更低运行圆轨道 (约 $100 \mathrm{~km}$ ) 的 “嫦娥” 2 号 $(\mathrm{CE}-2)$ 卫星也于 2010 年 10 月 1 日成功发射. “嫦娥” 1 号和 2 号, 在世界上首次搭载了 4 通道微波辐射计, 频率分别为 $3.0,7.8,19.35,37.0 \mathrm{GHz}$, 观 
察角度为 $0^{\circ}$, 其空间分辨率分别约为 $50 \mathrm{~km}(3 \mathrm{GHz})$ 和 $35 \mathrm{~km}$ (其余 3 通道), 测量精度为 $0.5 \mathrm{~K}^{[3]}$. 其 任务旨在测量整个月表面的微波辐射亮度温度 ( $\mathrm{Tb}$, brightness temperature), 进而反演月表层物理温 度和月壤层厚度, 估算月壤层富含的氦 $-3\left({ }^{3} \mathrm{He}\right)$ 含量 ${ }^{[3 \sim 12]}$.

本文用辐射传输理论分析了富有离散岩石块分布的新生环行山月球表面的微波热辐射, 以及红外 及微波热辐射变化的 “冷热点” 异常对比. 选取新生环行山 Tycho (第谷) 区域和相近纬度的老环行山 Maurolycus (莫洛利卡斯) 区域的 CE-1 微波辐射亮度温度 (Tb) 数据, 给出月表面微波热辐射的昼夜 变化. 以 3 层辐射传输模型为基础, 用 $\mathrm{CE}-1 \mathrm{~Tb}$ 数据反演了该两典型环形山区域的月尘层与月壤层的 物理温度分布及其昼夜变化. 结果表明: 主要由陨石撞击形成的新生环形山的微波与红外热辐射、及 其月表面物理温度的昼夜变化所表现出的异常分布, 与其大量离散分布高热传导率低热惯性岩石块月 貌密切相关.

\section{2 富有岩石块分布的新生环行山区域的热辐射}

由 Planck 辐射定理, 物体热发射强度为 ${ }^{[13]}$

$$
I=e \mu \varepsilon^{\prime} \frac{h v^{3}}{e^{h v / B T}-1} \equiv e F(v, T),
$$

其中 $e$ 是热射率, $T$ 是物体的物理温度, $v$ 是频率. 假定月表面由两部分组成: 具面积占空比 $f_{s}$ 的岩 石块, 其余为面积占空比 $\left(1-f_{s}\right)$ 的周围月表面. 这样, 月表面热辐射近似写成

$$
I=f_{s} e_{s} F_{s}\left(v, T_{s}\right)+\left(1-f_{s}\right) e_{f} F_{f}\left(v, T_{s}\right),
$$

其中下标 $s$ 与 $f$ 分别表示石块与周围月表面, 这里简单地忽略了石块与周围月表面辐射的相互作用.

由总辐射强度 $I$ 的测量, 可推算岩石块部分的热发射率为

$$
e_{s}=\frac{I-\left(1-f_{s}\right) e_{f} F_{f}\left(v, T_{s}\right)}{f_{s} F_{s}\left(v, T_{s}\right)}
$$

其中, 石块热辐射的昼夜变化可写为

$$
\Delta I=e_{s}\left[F_{s}\left(T_{s}^{d}\right)-F_{s}\left(T_{s}^{n}\right)\right],
$$

其中上标 $d$ 和 $n$ 分别表示白天与夜晚. 若是完全没有岩石块分布 (上标为) 的月表面, 其辐射的昼夜 变化写为

$$
\Delta I^{0}=e_{f}\left[F_{f}\left(T_{f}^{d}\right)-F_{f}\left(T_{f}^{n}\right)\right] .
$$

简便起见, 我们假定有两个月表面: 一个只有岩石块分布, 另一个没有, 新生环形山表面可以看作 是这两种表面的混合体, 而其中热辐射昼夜变化的异常主要由岩石引起. 因此, 我们只须讨论岩石表 面与没有岩石表面的热辐射变化, 以此对比新老环形山的热辐射差异, 这样式 (4) 与 (5) 之比写为

$$
\frac{\Delta I}{\Delta I^{0}}=\frac{e_{s}\left[F_{s}\left(T_{s}^{d}\right)-F_{s}\left(T_{s}^{n}\right)\right]}{e_{f}\left[F_{f}\left(T_{f}^{d}\right)-F_{f}\left(T_{f}^{n}\right)\right]}
$$

它给出了相近纬度上新生环行山表面的岩块和经历长年陨石冲击粉碎、乏有岩石块分布的老环 行山表面两者热辐射昼夜变化的差别. 
由互易性定理, 粗粘表面的热发射率计算为

$$
e_{p}(\theta, \varphi)=1-r_{p}(\theta, \varphi)=1-r_{p, \operatorname{coh}}(\theta, \varphi)-r_{p, \text { incoh }}(\theta, \varphi),
$$

其中 $r_{p}(\theta, \phi)$ 是 $p$ 极化反射率 $(p=v, h)$, 其相干部分 $r_{p, \mathrm{coh}}(\theta, \phi)$ 与非相干部分 $r_{p, \text { incoh }}(\theta, \phi)$ 分别计算 为 $[13,14]$

$$
\begin{aligned}
& r_{p, \operatorname{coh}}(\theta, \phi)=\left|R_{p}(\theta, \phi)\right|^{2} \exp \left(-4 \pi k^{2} \delta^{2} \cos ^{2} \theta\right), \\
& r_{p, \text { incoh }}(\theta, \phi)=\frac{1}{4 \pi} \int_{0}^{2 \pi} \mathrm{d} \phi^{\prime} \int_{0}^{\pi / 2} \mathrm{~d} \theta^{\prime} \sin \theta^{\prime}\left[\gamma_{p p}\left(\theta, \theta^{\prime} ; \phi-\phi^{\prime}\right)+\gamma_{p q}\left(\theta, \theta^{\prime} ; \phi-\phi^{\prime}\right)\right],
\end{aligned}
$$

其中 $R_{p}(\theta, \phi)$ 为粗粘面 $p$ - 极化反射系数, $k, \delta$ 分别为波数与粗粘面高度起伏方差. 可以看出, 相比平 表面, 粗䊁度 $\delta$ 使 $r_{p, \mathrm{coh}}$ 减小, 热发射率 $e_{p}$ 增加. 当频率与表面粗䊅度继续增加使得 $r_{p, \mathrm{coh}} \rightarrow 0$, 而使 非相干项 $r_{p, \text { incoh }}$ 占主要因素. 式 (8b) 的 $\gamma_{p q}$ 是粗䊅面 $p q-$ 极化 $(p, q=v$ or $h)$ 双站散射系数.

由于月表面反射率比较小 (月面介电常数 $\varepsilon \sim 3$ ), 可有热发射率 $e_{s} \sim e_{f}>0.8$, 以及 $e_{s} / e_{f} \sim 1$. 这 样式 (6) 的主要差别在于 $\left[F_{s}\left(T_{s}^{d}\right)-F_{s}\left(T_{s}^{n}\right)\right] /\left[F_{f}\left(T_{f}^{d}\right)-F_{f}\left(T_{f}^{n}\right)\right]$. 即是说, 新生环形山区域月表面辐射 的昼夜变化取决于岩石块与周围月表面热辐射昼夜变化之比.

在月蚀期间, 已有发现有大量的新生环行山其区域的红外热辐射要高于其四周的月表面, 在较好 的空间分辨率的红外辐射扫描图上表现为大量的 “热点” [1]. 在短暂的月蚀期, 这些辐射异常表现的 “热点” 主要来自于高热传导的岩石块物理温度要高于四周月表面环境.

由于红外的穿透深度几乎没有, 观测值直接就是月表面的物理温度表现. 在月球白天, 月表各处 达到热平衡后在同纬度地区有 $F_{s}\left(T_{s}^{d}\right) \approx F_{f}\left(T_{f}^{d}\right)$. 而在夜间, 由于岩石块热惯性大, 而保持热量能力较 好, 有 $T_{s}^{n}>T_{f}^{n}$, 而使得月表面 $F_{s}\left(T_{s}^{n}\right)>F_{f}\left(T_{f}^{n}\right)$. 这样使得式 (11) $\Delta I<\Delta I^{0}$, 即是: 具岩石块分布 的新生环行山表面红外辐射昼夜变化小于没有岩石块分布的老环行山区域.

在微波频段, 由 Rayleigh-Jeans 近似 $h v \ll B T$, 微波辐射亮度温度由式 (1) 定义为 ${ }^{[13,14]}$

$$
T_{b}=e T .
$$

这样, 式 (4) 的微波辐射亮度温度昼夜变化写为

$$
\begin{aligned}
& \Delta T_{b}=e_{s}\left(T_{s}^{d}-T_{s}^{n}\right), \\
& \frac{\Delta T_{b}}{\Delta T_{b}^{0}}=\frac{e_{s}\left(T_{s}^{d}-T_{s}^{n}\right)}{e_{f}\left(T_{f}^{d}-T_{f}^{n}\right)} .
\end{aligned}
$$

热惯性参数定义为获得与失去热量的能力:

$$
\gamma=\sqrt{\rho K C},
$$

其中 $\rho, K, C$ 分别为月壤的体密度、热传导率、热容量. 由于岩石的热惯性要比岩石四周的月表面大 得多, 保持热量的能力强, 因此在夜间或月蚀期间没有太阳光照时表现为热点. 然而, 由于微波的穿透 深度大, 在没有岩石块的情况下可以探测到下面的月壤层, 而月尘月壤层的物理温度廓线表现为随深 度白天由高变低、而夜晚则由低变高. 在夜间, 没有岩石块的月面辐射纳入了月壤下层较高温度的 $T$, 即式 (11) 中的 $T_{f}^{n}$ 是增加了, 导致有 $T_{s}^{n}<T_{f}^{n}$, 使得式 (11) 中 $\Delta T_{b}>\Delta T_{b}^{0}$, 即表现为 CE-1 观测到的 夜间微波辐射的 “冷点” ${ }^{[15]}$. 换言之, 岩石块环行山表面即使其物理温度昼夜差别较小, 但是微波热辐 射的昼夜差异却比周围要大. 


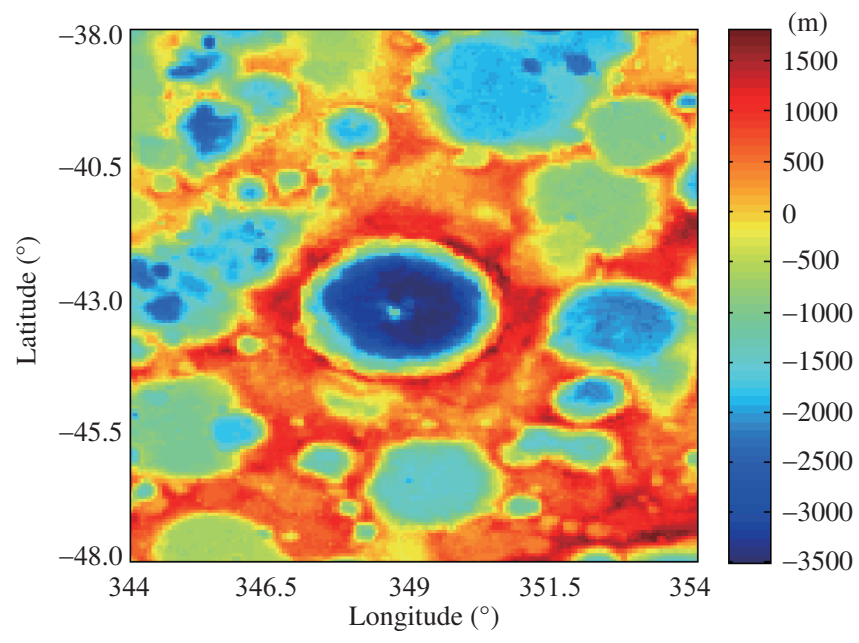

图 1 Tycho 环行山的地形图

Figure 1 Topography of the Tycho Crater

\section{3 嫦娥一号观测的典型环行山区域的辐射亮度温度昼夜变化}

作为例子, 选取新生的 Tycho 环行山 $\left(43^{\circ} \mathrm{S}, 349^{\circ} \mathrm{E}\right)$. Shorthill 等 [1] 曾用红外扫描图像发现了红 外辐射 “热点” 分布. 图 1 给出了 LOLA (lunar orbit laser altimeter) 测量到的 Tycho 环行山的地形 图. Tycho 环行山处在图中的中心, 右侧的图例以米为单位, 图的大小区域占经纬度 $10^{\circ} \times 10^{\circ}$, 成图分 辨率为每个像素 $0.1^{\circ}$ (以下的各图都取相同的大小、图例以及分辨率, 不另述), 实际上 LOLA 提供的 最高的分辨率可以达到每个经纬度 128 像素, 但是由于这样的数据量太大, 本文计算一般不需要.

图 2(a) (d) 分别给出 Tycho 环行山区域由 CE-1 37.0 与 $19.35 \mathrm{GHz}$ 通道观测的白天与晚上的辐 射亮度温度 $T_{\mathrm{b} 37}$ 与 $T_{\mathrm{b} 19}$. 可看出, 环行山区域白天微波热辐射比周围区域要强得多, 而夜晚环行山表 面微波热辐射要冷得多. 这种白天辐射 “热点”与晚上辐射 “冷点” 现象与文献 [15] 曾有过的讨论是 一致的. 文献 [15] 也曾指出这些微波辐射计观测到的 “冷点” 与红外观测到的夜间热点看来不一致, 但未曾进一步讨论.

与 Tycho 环行山成对比, 处在相近纬度的 Maurolycus 环行山是一个老年环行山, 应没有大量岩 石块的分布. 图 3 给出 Maurolycus 环行山的地形图. 由于两环行山纬度相近, 不至于由于纬度不同而 带来的物理温度太大的差异.

图 4(a) (d) 分别给出 Maurolycus 环行山表面由 CE-1 37.0 与 $19.35 \mathrm{GHz}$ 通道观测的白天与晚上 的辐射亮度温度. 可看出, 辐射亮度温度随纬度降低而有所增加. 在夜间, 也没有与 Tycho 环形山对应 的 “冷点” 出现.

可以看到, 岩石由于介电常数虚部大, 微波对其穿透深度小, 岩石起了遮挡月壤下层热辐射的作 用, 使得 Tycho 等新生环形山微波辐射的昼夜变化增大; 而 Maurolycus 等年老环形山由于不存在石 块, 其微波热辐射昼夜变化差异较小.

应指出的是, 通过对比 Diviner 红外辐射对两个环行山区域的观测, 红外与微波频段结果的差异 就会显现出来. 这主要是微波频段具有一定的穿透深度, 月壤下层温度对辐射有影响, 在这一深度内 存在的温度廓线造成了差异, 而红外频段只是观测了月表面.

图 5 和 6 分别给出了 Tycho 与 Maurolycus 环形山白天和黑夜 Diviner 红外辐射观测结果. 可以 

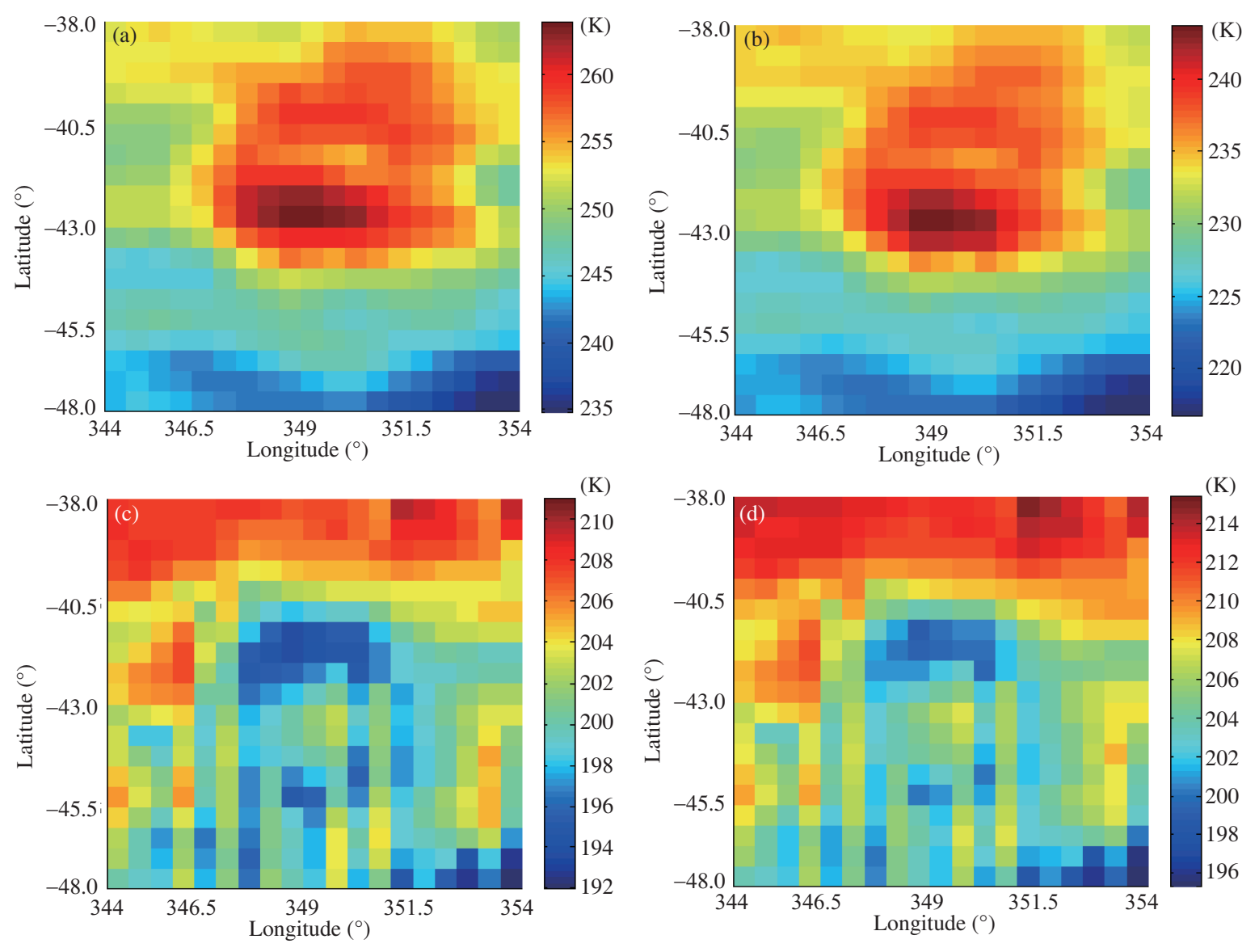

图 2 Tycho 环行山区域 CE-1 白天与晚上的 $T_{\mathrm{b} 37}$ 和 $\boldsymbol{T}_{\mathrm{b} 19}$

Figure 2 CE-1 data, $T_{\mathrm{b} 37}$ and $T_{\mathrm{b} 19}$, of the Tycho Crater at daytime and nighttime. (a) $T_{\mathrm{b} 37}$ at daytime; (b) $T_{\mathrm{b} 19}$ at daytime; (c) $T_{\mathrm{b} 37}$ at nighttime; (d) $T_{\mathrm{b} 19}$ at nighttime

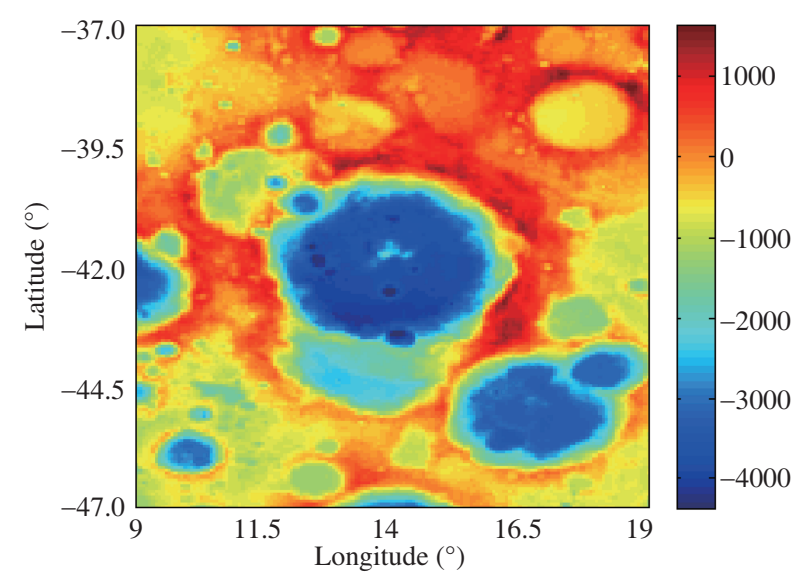

图 3 Maurolycus 环行山的地形图

Figure 3 Topography of Maurolycus Crater 

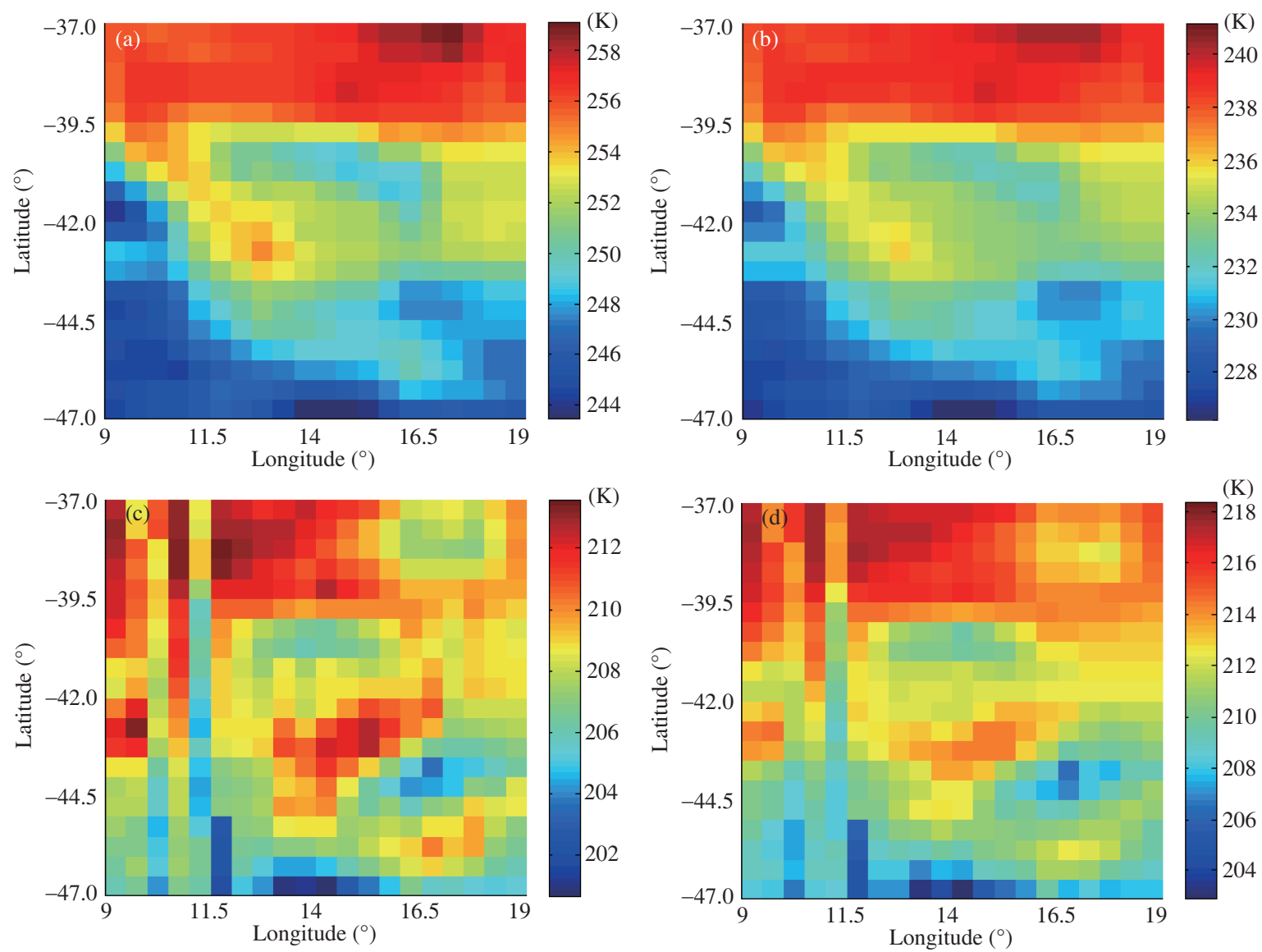

图 4 Maurolycus 环行山区域 CE-1 观测白天与晚上的 $T_{\mathrm{b} 37}$ 和 $T_{\mathrm{b} 19}$ 数据

Figure 4 CE-1 data, $T_{\mathrm{b} 37}$ and $T_{\mathrm{b} 19}$, of the Maurolycus Crater at day and nighttime. (a) $T_{\mathrm{b} 37}$ at daytime; (b) $T_{\mathrm{b} 19}$ at daytime; (c) $T_{\mathrm{b} 37}$ at nighttime; (d) $T_{\mathrm{b} 19}$ at nighttime
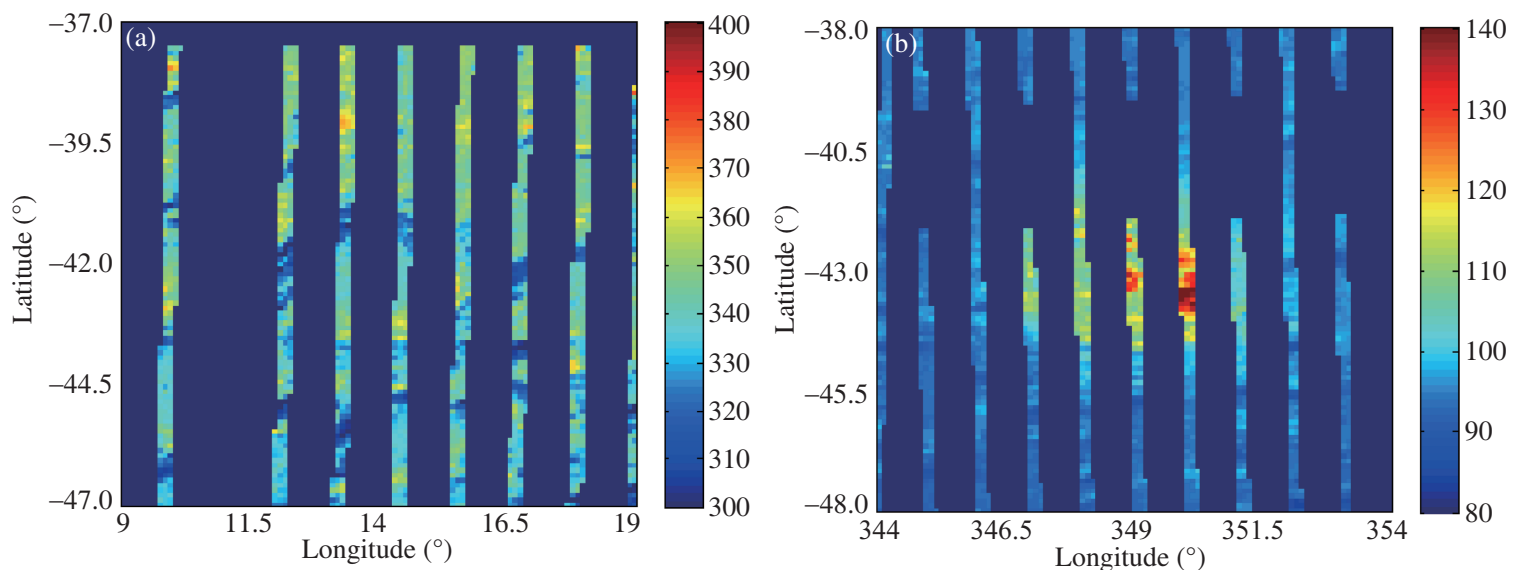

图 5 Diviner 红外辐射计第 8 通道通道观测到的 Tycho 地区的热辐射

Figure 5 The IR thermal emission of Tycho Crater observed from Diviner Channel 8. (a) At daytime (around 13:00); (b) at nighttime (around 0:00), when "hot spots" can be seen 

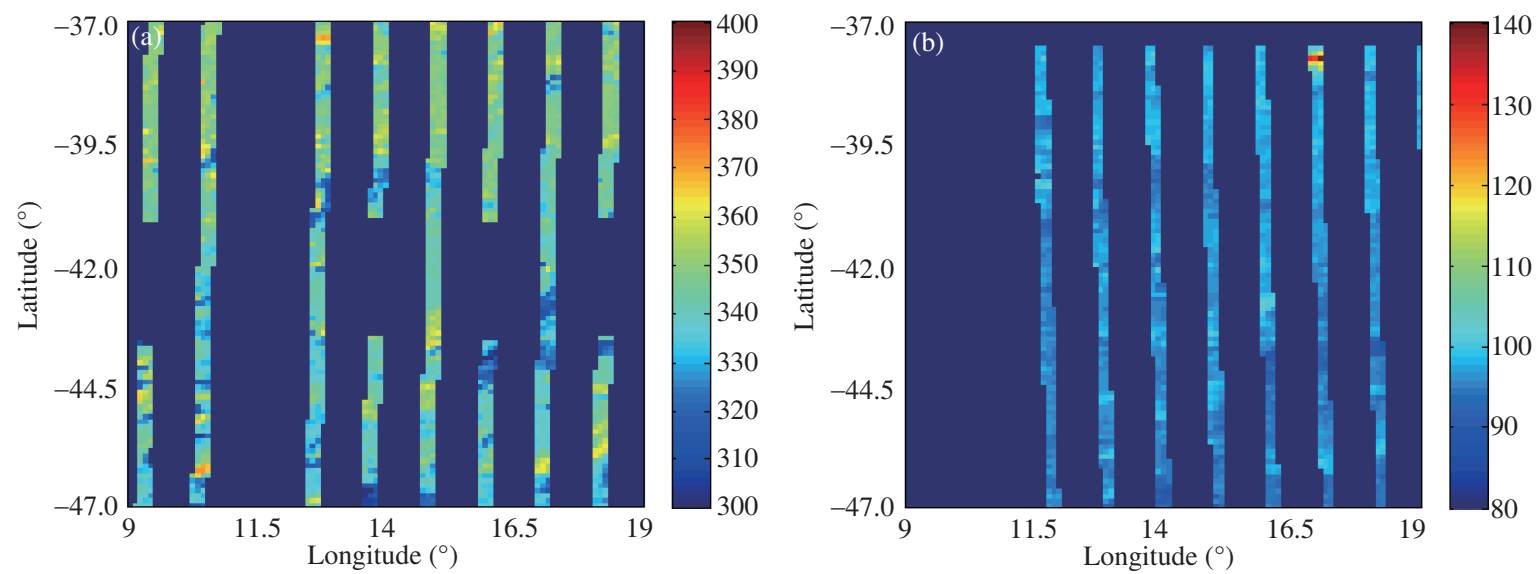

图 6 Diviner 红外辐射计第 8 通道观测到的 Maurolycus 地区的热辐射

Figure 6 The IR thermal emission of the Maurolycus Crater observed from Diviner Channel 8. (a) At daytime (around 13:00); (b) at nighttime (around 23:00)
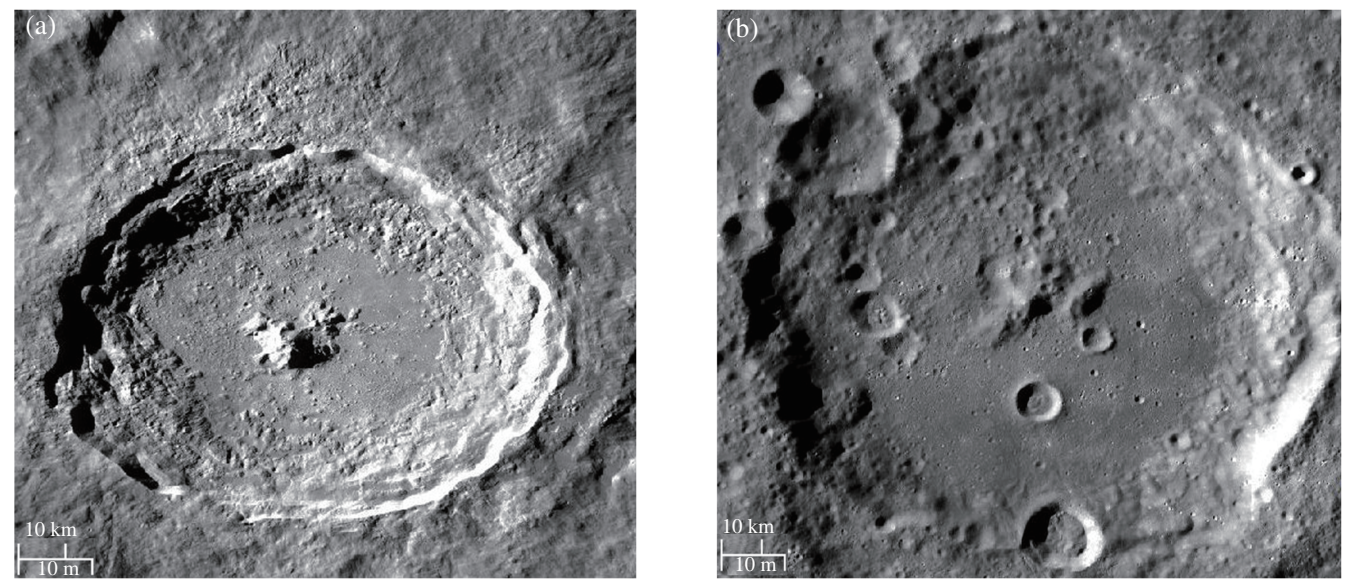

图 7 (a) Tycho 与 (b) Maurolycus 环形山由 LROC 拍摄到的光学图像

Figure 7 Optical images of (a) Tycho and (b) Maurolycus Craters from LROC

看到, 在白天由于太阳光持续照射, 月表面温度达到热平衡后, Tycho 与 Maurolycus 环形山红外辐射 温度分布都只与地形与太阳光照有关: 朝向赤道的一面温度较高, 朝向极地背离太阳光照射的一面温 度较低. 但是, 在夜间, 红外辐射结果表明 Tycho 环形山的红外辐射要比周围高, 即是所谓的 “热点”, 而 Maurolycus 环形山红外辐射与周围环境没有明显差异. 可见, Tycho 等新生环形山的红外辐射昼夜 差要比 Maurolycus 等年老环形山小, 但是在微波频段却恰恰相反.

图 7 给出了 LROC (lunar reconnaissance orbiter camera) 拍摄的 Tycho 和 Maurolycus 环形山 的光学图像 (http://lroc.sese.asu.edu/), 在 Tycho 环形山内部可以清晰的看到大量岩块的存在, 而在 Maurolycus 环形山很难发现离散的岩块.

我们用月壤物理温度廓线对月表面热辐射的影响, 来阐述新生环形山与年老环形山红外与微波辐 射的昼夜变化. 首先, 图 8(a) 在 Tycho 新生环形山中, 由于石块的热传导性好, 它的存在延伸了月表层 的物理温度. 图中两个黑色的条块分别表示在白天和黑夜微波辐射计探测到的岩石的物理温度范围, 在白天, 月表面石块的物理温度与没有岩石的周围环境是相同的, 但是在夜晚却要比周围高大约 $40 \mathrm{~K}$. 由于岩石介电常数的虚部大微波穿透深度在有岩石的地方比较小; 当岩块较大时, 微波根本穿透不过 

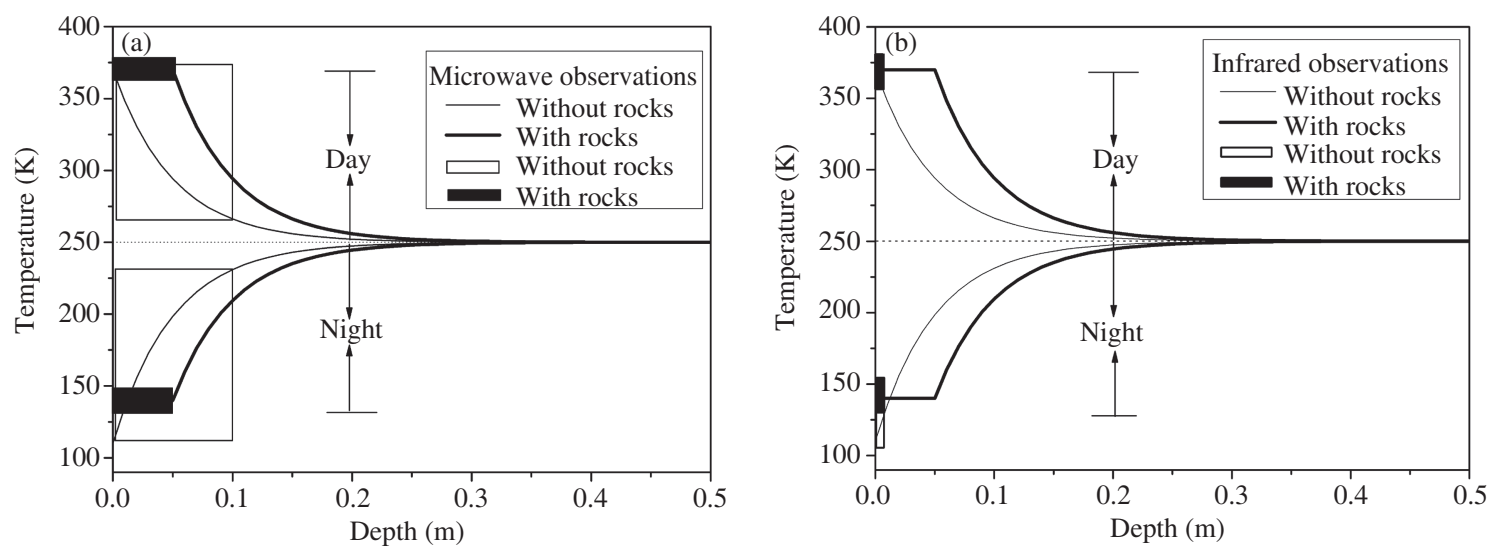

图 8 新生环形山与年老环形山的 (a) 微波与 (b) 红外辐射观测示意图 (温度廓线的选择根据文献 [9])

Figure 8 A model illustration of (a) MW and (b) IR emissions from fresh (with rocks) and old (free of rocks) craters in day and night temperature profiles. The temperature profile is proposed by Vasavada et.al [9] using a two layer model 岩石.

图 8(a) 中的两个空白方框表示微波在没有岩石区域所探测到的物理温度范围, 可以看到, 在夜晚, 尽管岩石的物理温度比周围环境高, 但是由于下垫月壤层介质的温度较高 (约为 $250 \mathrm{~K}$ ), 没有岩石区 域时下垫月壤层的贡献使得总的微波辐射可能高于仅靠岩块热辐射贡献的岩石区域. 即是说, 新生环 形山微波热辐射的昼夜差异要高于年老环形山, 这就是在没有太阳光照射情况下微波观测的 “冷点”. 图 8(a) 中所示的月表的物理温度值是根据 Diviner 的观测结果, 月壤层的物理温度假定为 $250 \mathrm{~K}^{[16]}$.

然后, 由图 8(b) 可看到, 红外频段几乎没有穿透能力, 它所探测到的热辐射只来自于月表面, 完全 没有来自下垫月壤层的贡献, 在图 8 中由细条块和细方框表示. 在月球黑夜, 由于岩石的存在的地方 物理温度高, 红外热辐射强, 这就是夜晚观测到的红外辐射 “热点” .

\section{4 典型环行山区域的物理温度昼夜变化}

由 3 层辐射传输模型, $0^{\circ}$ 观测角的辐射亮度温度写为 ${ }^{[5]}$

$$
\begin{aligned}
T_{\mathrm{b}}\left(0^{\circ}\right)= & \frac{k_{0} \varepsilon_{1}^{\prime \prime}}{2 \varepsilon_{0} k_{1}^{\prime \prime}}\left|X_{01}\right|^{2}\left(1-e^{-2 k_{1}^{\prime \prime} d_{1}}\right)\left(1+\left|R_{12}\right|^{2} e^{-2 k_{1}^{\prime \prime} d_{1}}\right) T_{1} \\
& +\frac{k_{0} \varepsilon_{2}^{\prime \prime}}{2 \varepsilon_{0} k_{2}^{\prime \prime}}\left|X_{01} X_{12}\right|^{2}\left(1-e^{-2 k_{2}^{\prime \prime} d_{2}}\right)\left(1+\left|R_{23}\right|^{2} e^{-2 k_{2}^{\prime \prime} d_{2}}\right) e^{-2 k_{1}^{\prime \prime} d_{1}} T_{2} \\
& +\frac{k_{0} \varepsilon_{3}}{2 \varepsilon_{0} k_{3}^{\prime \prime}}\left|X_{01} X_{12} X_{23}\right|^{2} e^{-2 k_{1}^{\prime \prime} d_{1}} e^{-2 k_{2}^{\prime \prime} d_{2}} T_{3},
\end{aligned}
$$

其中, $\varepsilon_{n}(n=0,1,2,3)$ 与 $k_{n}(n=0,1,2,3)$ 分别为自由空间、介质层 1 (尘土层)、 2 (月壤)、 3 (岩床) 的 介电常数与波数. 上标” 表示虚部, $d_{1}$ 与 $d_{2}$ 分别是月表面尘土层与月壤层的厚度. 参数 $X_{n m}$ 与 $R_{n m}$ 分别是三层模型中分层界面 $n m(n, m=0,1,2,3)$ 的透射与反射系数.

月表红外与微波热辐射观测与反演的主要区别在于微波具有一定的穿透深度, 并且月壤层有温度 分布廓线, 而红外仅能观测到月表层的热辐射. 已有文献 [9 12], 尘土层与月壤层的物理温度 $T_{1}$ 与 $T_{2}$ 可由高频率通道的辐射亮度温度 $T_{\mathrm{b} 19}$ 与 $T_{\mathrm{b} 37}$ 反演得到. 由于高频率通道对月壤与岩床的界面不再敏 感, 于是令 $d_{2} \rightarrow \infty$, 得到 

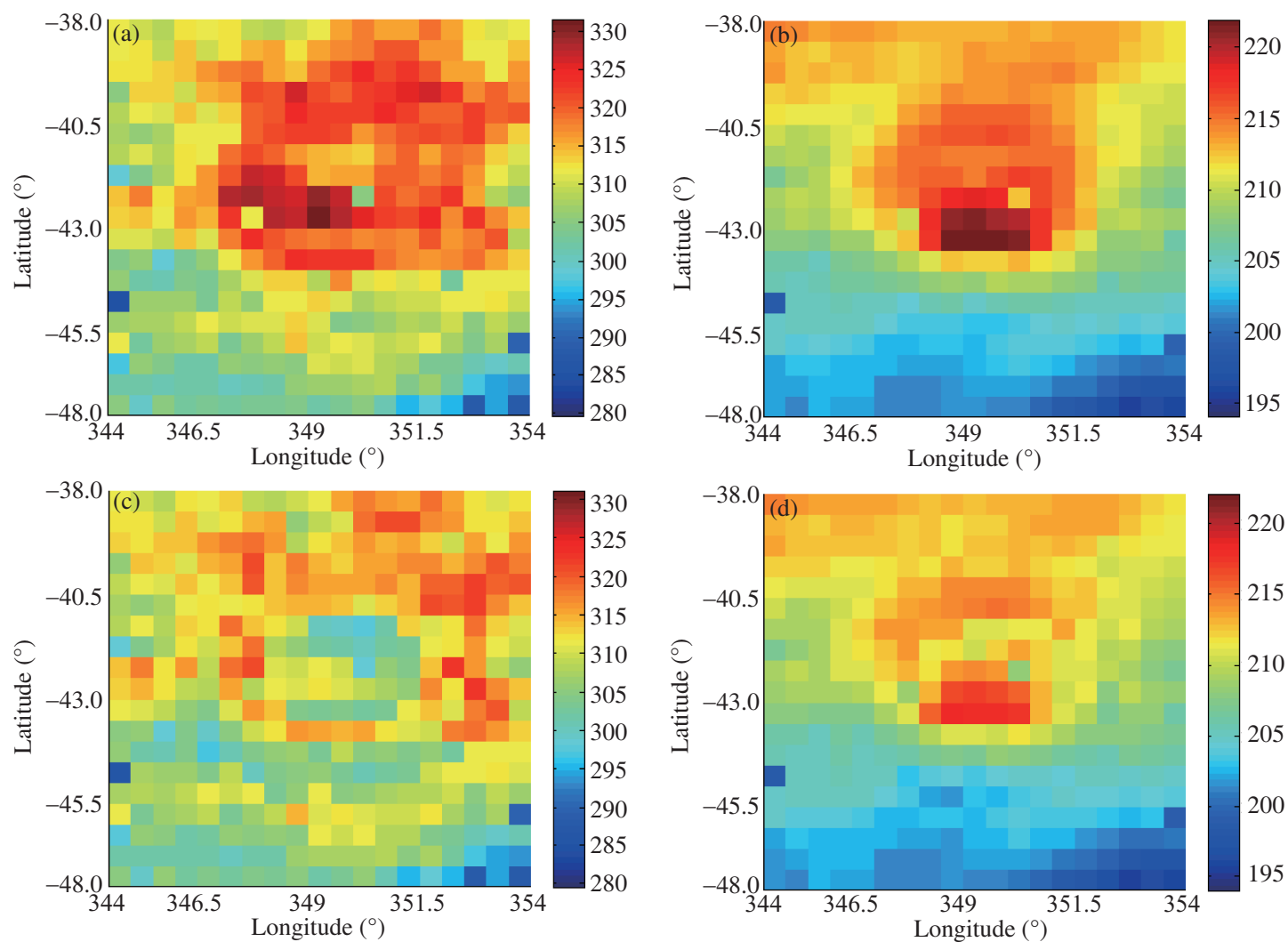

图 9 白天 Tycho 环行山月尘层和月壤层反演的物理温度 $T_{1}$ 与 $T_{2}$

Figure 9 At daytime, inverted of the Tycho Crater (a) $T_{1}$ and (b) $T_{2}$ without rocks; (c) $T_{1}$ and (d) $T_{2}$ with rocks on surface

$$
\begin{gathered}
T_{1}=\frac{B_{37} T_{\mathrm{b} 19}-B_{19} T_{\mathrm{b} 37}}{A_{19} B_{37}-A_{37} B_{19}}, \quad T_{2}=\frac{A_{37} T_{\mathrm{b} 19}-A_{19} T_{\mathrm{b} 37}}{A_{37} B_{19}-A_{19} B_{37}}, \\
A_{\nu}=\left(1-r_{01}\right)\left(1-e^{-\alpha_{1} \nu d_{1}}\right)\left(1+r_{12} e^{-\alpha_{1} \nu d_{1}}\right), \quad B_{\nu}=\left(1-r_{01}\right)\left(1-r_{12}\right) e^{-\alpha_{1} \nu d_{1}}
\end{gathered}
$$

$\alpha_{1}$ 表示吸收系数, $\nu$ 为频率 $(\nu=19.35,37.0 \mathrm{GHz}), d_{1}$ 为月尘层厚度, 计算中取 $d_{1}=0.2 \mathrm{~m}^{[8]}$.

月层介质的成分 (如 $\mathrm{FeO}+\mathrm{TiO}_{2}$ 含量等) 决定了其介电常数, 影响发射率和辐射亮度温度. 设月壤 每一分层都是均匀的, 其介电常数为 $\varepsilon=\varepsilon^{\prime}+i \varepsilon^{\prime \prime}$.

由文献 [14] 可知 $\varepsilon$ 计算为:

$$
\begin{gathered}
\varepsilon^{\prime}=2.75 \pm 0.20\left(\text { as } \rho=1.7 \mathrm{~g} / \mathrm{cm}^{3}\right), \\
\log _{10}(\tan \delta)=-2.395(0.052)+0.064(0.015) \times \mathrm{TiO}_{2} \quad\left(\text { as } \rho=1.7 \mathrm{~g} / \mathrm{cm}^{3}\right) .
\end{gathered}
$$

当孔隙率为时, 月壤的体积密度 $\rho$ 即为比重, 通常写作 $\rho_{0}\left(\mathrm{~kg} / \mathrm{m}^{3}\right)=27.3 \times \mathrm{FeO}+11.0 \times \mathrm{TiO}_{2}+2773$ 对于其他给定的体积密度 $\langle\rho\rangle$, 其对应的介电常数 $\langle\varepsilon\rangle$ 可由 Maxwell-Garnett 公式变换得到:

$$
\frac{1}{\langle\rho\rangle} \frac{\langle\varepsilon\rangle-1}{\langle\varepsilon\rangle+2}=\frac{1}{\rho} \frac{\varepsilon-1}{\varepsilon+2} .
$$

一般地, 月尘层的孔隙率假定为 0.58 , 月壤层为 $0.42^{[17]}$.

结合月壤的孔隙率, 计算月壤有效介电常数 $\varepsilon_{1}(z)=\varepsilon_{g}\left(=\varepsilon_{g}^{\prime}+i \varepsilon_{g}^{\prime \prime}\right), \alpha_{1}=(2 \pi / \mathrm{c})\left(\varepsilon_{g}^{\prime \prime} / \sqrt{\varepsilon_{g}^{\prime}}\right)$, 其中 $\mathrm{c}$ 

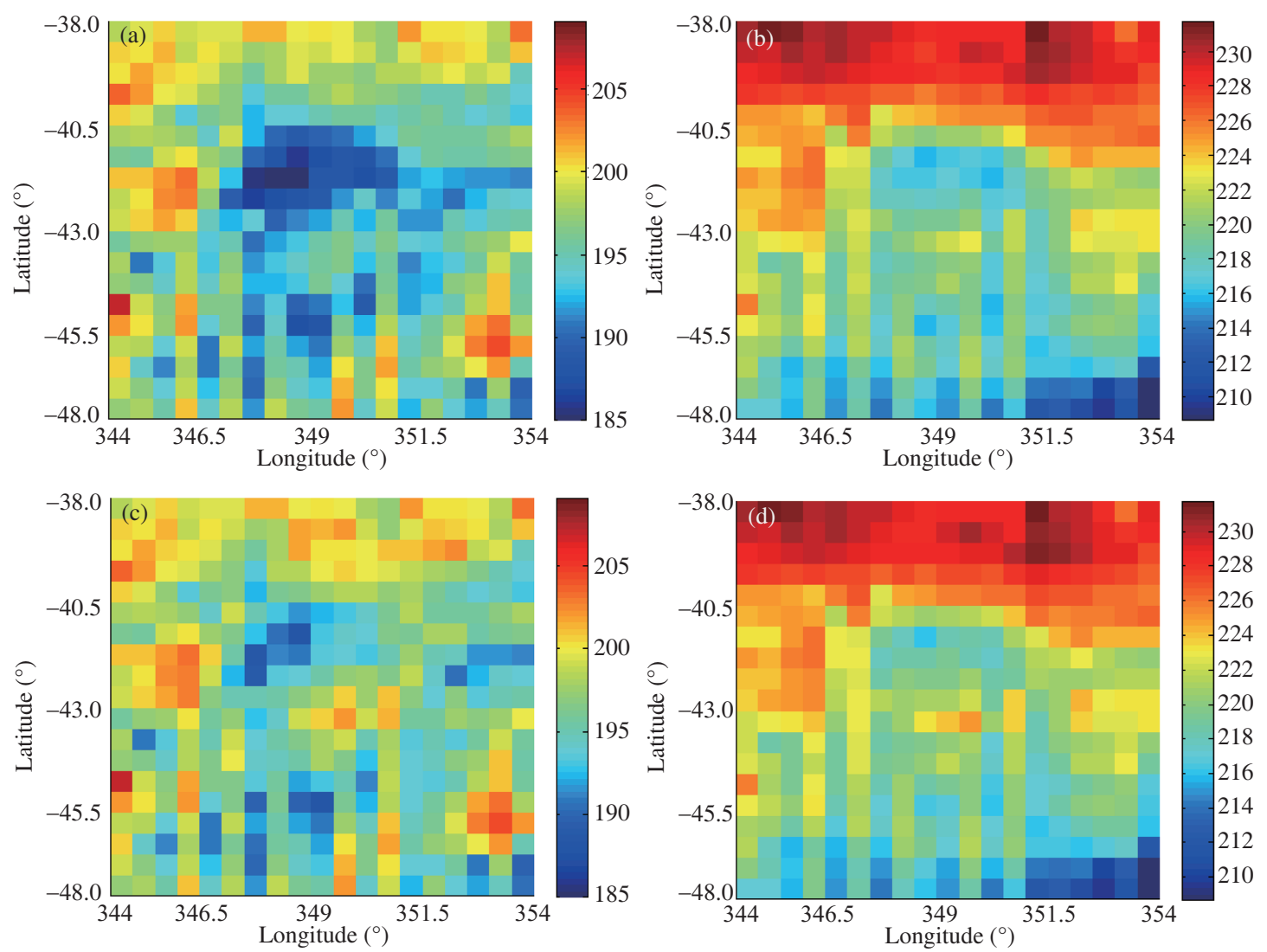

图 10 夜间 Tycho 环行山月尘层和月壤层反演的物理温度 $T_{1}$ 与 $T_{2}$

Figure 10 At night time, the inverted (a) $T_{1}$ and (b) $T_{2}$ without rocks; (c) $T_{1}$ and (d) $T_{2}$ with rocks on surface of the Tycho Crater

为真空中光速, $\varepsilon_{g}$ 视为月壤有效介电常数, 主要由月壤体积密度、 $\mathrm{FeO}$ 和 $\mathrm{TiO}_{2}$ 含量来决定. 将 $\alpha_{1}$ 和 其他已知参数代入式 (14) 和 $(15)$ 由 $\mathrm{Tb}_{37}$ 和 $\mathrm{Tb}_{19}$ 反演得到月尘层 $T_{1}$ 与月壤层 $T_{2}$.

新生环形山岩石块的存在改变了月表面的介电常数和热惯性, 同时也影响了微波的穿透深度. 为 表明岩石对物理温度 $T_{1}$ 和 $T_{2}$ 反演的影响, 先假设在 Tycho 环形山表面没有岩石存在. 图 9(a) 和 (b) 给出了白天 Tycho 区域假设没有岩石时, 其 $T_{1}$ 和 $T_{2}$ 的反演结果, 可以看到反演的 $T_{1}$ 分布与微波 $T_{\mathrm{b} 37}$ 和 $T_{\mathrm{b} 19}$ 分布类似, Tycho 环行山内要比周围区域温度高.

根据 Bandfield 等 ${ }^{[2,18]}$ 对 Tycho 环行山内岩石丰度的计算, 环形山内平均的岩石面积占空比 $f_{\text {rock }}$ 取为 0.03 , 在环形山边缘取为 0.01 , 远离环形山的地方取为, 这些区域的划分是参考 Tycho 环形山地 形图与文献 [17] 的计算结果确定的. 岩石的介电常数取为 $\varepsilon_{\text {rock }}=6.0+i 0.1^{[17,18]}$, 这样在存在岩块的 区域, 其平均介电常数可以写为 $\left\langle\varepsilon_{1}\right\rangle=f_{\text {rock }} \varepsilon_{\text {rock }}+\left(1-f_{\text {rock }}\right) \varepsilon_{\text {soil }}$.

图 9 (c) 和 (d) 给出有岩石存在时 Tycho 区域白天 $T_{1}$ 和 $T_{2}$ 的反演结果. 可以看到, 环形山内的 $T_{1}$ 比没有岩石时的 $T_{1}$ 降低了, 这结果与红外观测是一致的, 如图 6(a) 中所示, 环形山红外辐射温度 与周围区域类似. 在白天, 微波辐射来自于温度高的月表层和温度渐降的月壤层, 岩石占的比例大, 对 于一定的 $\mathrm{Tb}, T_{1}$ 就会低一些. 该环形山白天物理温度 $T_{1}$ 与周围区别不大, 也说明不是受可能的火山 口地热的影响.

在月球夜间, Tycho 区域 $T_{1}$ 和 $T_{2}$ 反演结果分别如图 10 (a) 和 (b) 和图 10 (c) (d) 所示. 反演得 

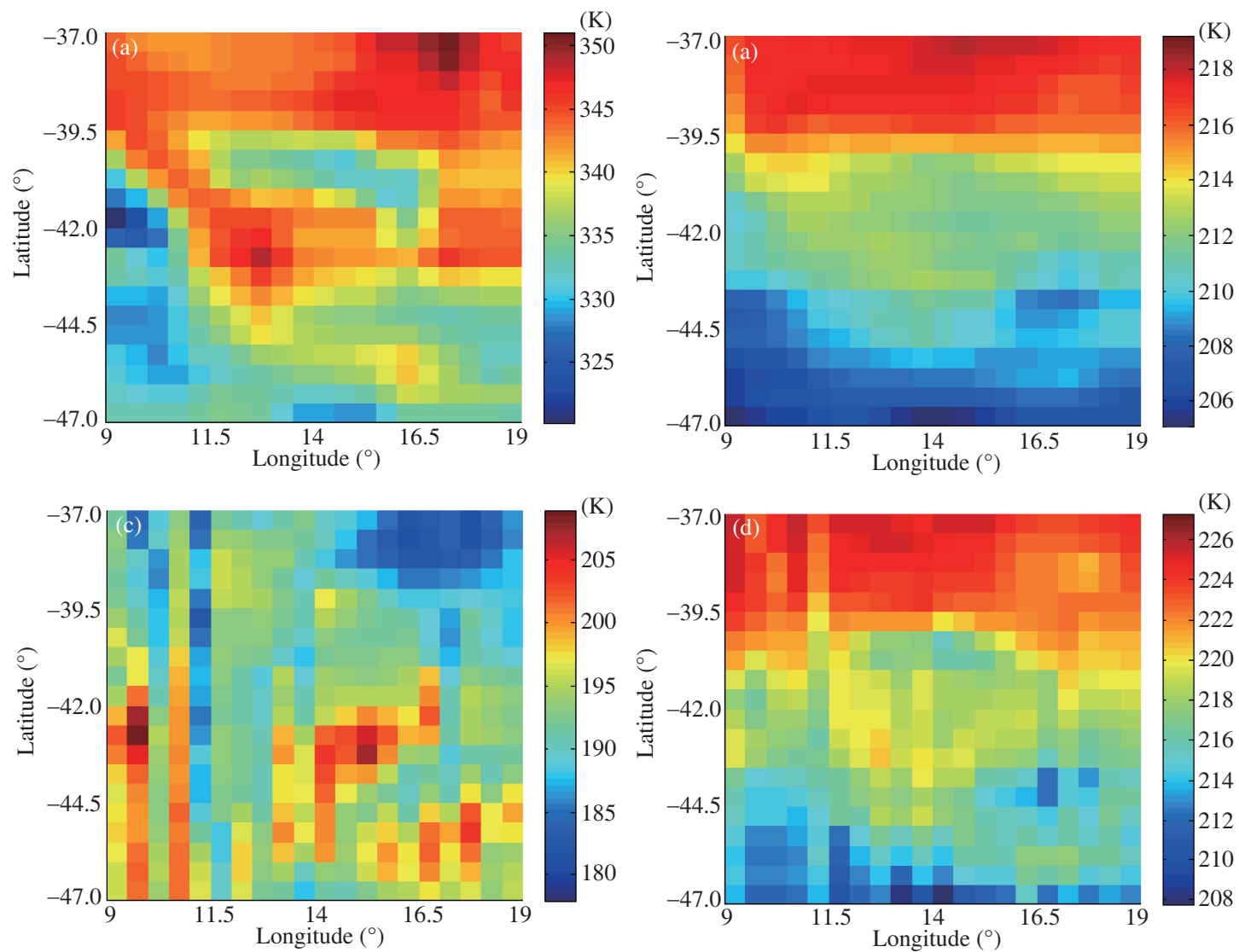

图 11 Maurolycus 环行山白天和黑夜月尘层和月壤层物理温度 $T_{1}$ 与 $T_{2}$

Figure 11 Inverted of Maurolycus crater. (a) $T_{1}$ and (b) $T_{2}$ at daytime; (c) $T_{1}$ and (d) $T_{2}$ at nighttime

到的环形山内月表层 $T_{1}$ 似乎并不像图 5 (b) 红外观测结果那么亮, 这是由于反演得到的 $T_{1}$ 是在一定 穿透深度内的平均结果. 由于夜间月壤温度廓线随深度递增, 无石块区域的微波辐射纳入了下垫月壤 层辐射贡献, 则使得该区域的 $T_{1}$ 升高, 这样使得环形山内外物理温度不会有太大的区别, 如图 8 (a) 所解释.

因此, 我们可以得到第二个结论: 新生环形山表层物理温度的昼夜变化不如年老环形山明显, 也 就是 $\Delta T_{1 \text { (fresh) }}<\Delta T_{1 \text { (old) }}$. 作为对照试验, 图 11(a) 和 (d) 分别给出了 Maurolycus 环形山白天和夜间 反演得到的 $T_{1}$ 和 $T_{2}$. 由于年老环形山中没有岩石来改变微波的穿透深度与影响月面介电常数, 因此 没有 “冷点” 和 “热点” 的异常现象出现. 此外可看到, 在图 11 (a) 和 (c) 的右上角, 正好有一个新生 的 Buch (布赫) 环形山, 但是, 由于它未有考虑石块存在, 使得反演的物理温度 $T_{1}$ 与 Tycho 未考虑岩 石时的反演结果类似. 在考虑 Buch 环形山有石块存在时, 应能得到与 Tycho 的 CE-1 微波反演值和 Diviner 红外观测值相一致的结果.

\section{5 结论}

选取富有离散岩石块分布的新生环行山 Tycho 区域与不再具有岩石块分布的老环行山 Maurolycus 区域的中国 CE-1 微波辐射观测数据, 显示该两典型环形山区域具有不同的微波辐射昼夜变化. 作 
为对比, 由 LRO Diviner 对上述两环行山区域的红外辐射观测结果, 以辐射传输为基础, 讨论了月表 面环形山红外与微波辐射的比较. 结果显示, 在如同 Tycho 的富有石块分布的新生环形山中, 微波热 辐射的昼夜变化要比年老环形山 (如 Maurolycus) 要明显得多, 这就是 CE-1 观测到的夜间环行山内 微波辐射“冷点”。这是由于夜间月壤层逆温廓线微波辐射为富有岩石的表层遮蔽引起的.

由三层辐射传输模型, 用 CE-1 高频通道的辐射亮度温度数据反演月面月尘层与月壤层的物理温 度, 结果表明: 新生环行山内物理温度昼夜变化较小. 这与只纳入月表面辐射的 Diviner 红外辐射观测 的 “热点” 是一致的.

月表面红外与微波辐射观测的 “冷点” 与 “热点”, 以及热辐射和月球表层物理温度与环形山内岩 石分布密切相关, 它引起月表层介电常数的变化、微波穿透深度和月表层热辐射总贡献的变化.

致谢 法文哲博士关于本文的工作给予了许多有意义的建议, 在此致谢.

\section{参考文献}

1 Shorthill R W, Saari J M. Nonuniform cooling of the eclipsed moon: A listing of thirty prominent anomalies. Science, 1965, 150: 210-212

2 Bandfield J L, Ghent R R, Vasavada A R, et al. Mapping lunar surface rock abundance and regolith thermalphysical properties using LRO Diviner data. In: 41st Lunar and Planetray Science Conference, 2010

3 Jiang J S, Jin Y Q. Selected Paper on Microwave Exploration of Lunar Surface in Chinese Chang'E-1 Project. Beijing: Science Press, 2011

4 Fa W, Jin Y Q. Quantitative estimation of helium-3 spatial distribution in the lunar regolith layer. Icarus, 2007, 71: $15-23$

5 Jin Y Q, Fa W. An inversion approach for lunar regolith layer thickness using optical albedo data and microwave emission simulation. Acta Astronaut, 2009, 65: 1409-1423

6 Fa W, Jin Y Q. Simulation of brightness temperature of lunar surface and inversion of the regolith layer thickness. J Geophys Res Planets, 2007, 112: 1-13

7 Gong X, Jin Y Q. Diurnal physical temperature at Sinus Iridum area retrieved from observations of CE-1 microwave radiometer. Icarus, 2012, 218: 807-816

8 Fa W, Jin Y Q. A primary analysis of microwave brightness temperature of lunar surface from Chang-E 1 multi-channel radiometer observation and inversion of regolith layer thickness. Icarus, 2010, 207: 605-615

9 Fa W Z, Jin Y Q. Quantitative estimation of He-3 spatial distribution in the lunar regolith layer. Sci China Ser D-Earth Sci, 2008, 38: 167-176 [法文哲, 金亚秋. 月球表面月壤中 ${ }^{3} \mathrm{He}$ 含量分布的定量估算. 中国科学 D 辑: 地球科学, 2008, 38: $167-176]$

10 Fa W Z, Xu F, Jin Y Q. SAR imaging simulation for an inhomogeneous undulated lunar surface based on triangulated irregular network. Sci China Ser F-Inf Sci, 2009, 39: 185-198 [法文哲, 徐丰, 金亚秋. 基于不规则三角网格剖分的非均匀 起伏月球表面 SAR 成像模拟. 中国科学 F 辑: 信息科学, 2009, 39: 185-198]

11 Jin Y Q, Fa W Z. The modeling analysis for microwave emission from stratified media of non-uniform lunar cratered terrain surface in Chinese Chang-E 1observation. Chinese Sci Bull, 2010, 55: 2040-2046 [金亚秋, 法文哲. 嫦娥 1 号对 环形山起伏非均匀月表层微波辐射观测的理论建模分析. 科学通报, 2010, 55: 2040-2046]

12 Gong X H, Jin Y Q. Diurnal distribution of the physical temperature at Sinus Iridum area retrieved from observations of CE-1 microwave radiometer. Chinese Sci Bull, 2011, 56: 1877-1886 [宫晓㥶, 金亚秋. 嫦娥一号微波辐射计观测月球虹 湾地区表面物理温度昼夜时间分布. 科学通报, 2011, 56: 1877-1886]

13 Jin Y Q. Electromagnetic Scattering Modelling for Quantitative Remote Sensing. Singapore: World Scientific, 1994

14 Tsang L, Kong J A, Shin R. Microwave Remote Sensing Theory. New York: John Wiley, 1985

15 Chan K L, Tsang K T, Kong B, et al. Lunar regolith thermal behavior revealed by Chang'E-1 microwave brightness temperature data. Earth Planet Sci Lett, 2010, doi:10.1016/j.epsl.2010.04.015 
16 Vasavada A R, Paige D A, Wood S E. Near-surface temperature on Mercury and the Moon and the stability of polar ice deposits. Icarus, 1999, 141: 179-357

17 Heiken G H, Vaniman D T, French B M. Lunar Source-Book: A User's Guide to the Moon. London: Cambridge University Press, 1991

18 Bandfield J L, Ghent R R, Vasavada A R, et al. Lunar surface rock abundance and regolith fines temperatures derived from LRO Diviner Radiometer data. J Geophys Res Planets, 2011, 116, E00H02, doi: 10.1029/2011JE003866

\title{
Diurnal change of thermal emission with "hot spots" and "cold spots" of fresh lunar craters observed by Chinese Chang'E-1
}

\author{
GONG XiaoHui \& JIN YaQiu* \\ Key Laboratory of Wave Scattering and Remote Sensing Information, Fudan University, Shanghai 200433, China \\ *E-mail: yqjin@fudan.edu.cn
}

\begin{abstract}
Hundreds of abnormally "hot spots" on lunar surface had been revealed by infrared scanning image during totality using grounded infrared (IR) scanner in the early 1960's, which turned out to be "cold spots" on the microwave (MW) brightness temperature distribution at night according to the Chang'E (CE)-1 radiometer observations. In this paper, a theoretical analysis of radiative transfer is presented to explain this abnormal phenomenon of diurnal changes. Two craters, typically representing the abnormal fresh craters rich in rock abundance and an old one almost with rock free, respectively, and located at similar latitudes are chosen for comparison of their diurnal temperature change. Using the measurements of CE-1 multi-channel microwave radiometer, brightness temperature distributions of these two craters are presented and applied to inversions of the physical temperatures of lunar regolith media, based on a three-layer radiative transfer model. The correlation between the diurnal MW and IR thermal changes of the lunar surface and the rock abundance is discussed.
\end{abstract}

Keywords CE-1 Tb, "hot spots" and "cold spots", thermal diurnal change, MW and IR emission, rock abundance, microwave, infrared radiation, planetary surface analysis

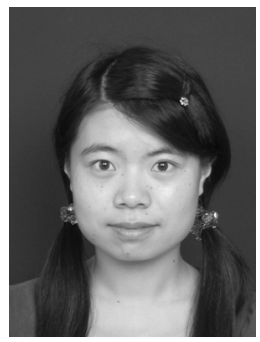

GONG XiaoHui was born in 1985 She received the B.S. degree from EastChina Normal University in 2008, and is now a graduate student pursuing the Ph.D. degree in Fudan University. Her research areas include microwave remote sensing and image/data processing for lunar exploration, inversions of lunar surface parameters, e.g., regolith layer structure and dielectric properties.

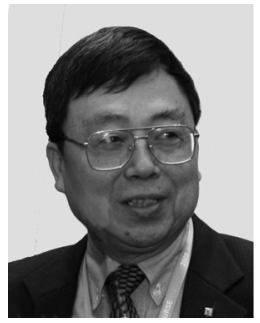

JIN YaQiu was born in 1946, the TePin Professor and Director of Key Laboratory of Wave Scattering and Remote Sensing Information, Fudan University. He received the $\mathrm{Ph}$.D. degree from Massachusetts Institute of Technology (MIT) in 1985, and he is IEEE Fellow, Academician of Chinese Academy of Sciences, and Principle Scientist of the State 973 Project in China. His research interests are electromagnetic scattering and radiative transfer, microwave remote sensing and computational electromagnetic. 